\title{
Perfil do desenvolvimento motor em escolares com excesso de peso
}

\section{Profile of the motor development in children with overweight}

\author{
Mayara Moura Alves da Cruz¹, Stephanie Dornelas Cesario², Caique Aguiar dos Santos², João Pedro Lucas Neves Silva², \\ Francis Lopes Pacagnelli², Deborah Cristina Gonçalves Luiz Fernani² \\ "Fisioterapia Aplicada à Cardiologia, Universidade Estadual Paulista "Júlio de Mesquita Filho" (UNESP) - Presidente Prudente (SP), Brasil. \\ ${ }^{2}$ Curso de Fisioterapia, Universidade do Oeste Paulista (UNOESTE) - Presidente Prudente (SP), Brasil. \\ '2Cús-graduação em Ciências, Universidade de São Paulo (USP) - São Paulo (SP), Brasil. \\ ${ }^{4}$ Pós-graduação em Ciências, Universidade Federal de São Paulo (UNIFESP) - São Paulo (SP), Brasil.
} Íbis Ariana Peña de Moraes ${ }^{3}$, Thais Massetti ${ }^{3}$, Talita Dias da Silva ${ }^{4}$, Carlos Bandeira de Mello Monteiro ${ }^{3}$, Maria Tereza Artero Prado²,

DOI: http://dx.doi.org/10.7322/abcshs.v42i3.1071

\section{RESUMO}

Introdução: Diferenças relacionadas ao peso e às habilidades motoras em crianças em idade escolar ainda apresentam controvérsias. Objetivo: Analisar a relação entre desenvolvimento motor e nível de atividade física com o índice de massa corporal (IMC) de escolares com excesso de peso. Métodos: Foram avaliados 85 escolares de ambos os sexos, de 6 a 10 anos, de Presidente Prudente, São Paulo, Brasil. Os instrumentos utilizados foram a classificação do IMC/idade, a Escala de Desenvolvimento Motor e - Questionário do Nível de Atividade Física Modificado e Adaptado. A amostra foi dividida em dois grupos: grupo com excesso de peso (GEP), com 50 escolares com diagnóstico nutricional de sobrepeso e obesidade; e grupo com normopeso (GNP), com 35 escolares eutróficos. Para análise dos dados obtidos foram utilizados: Shapiro Wilk (normalidade), teste $t$ de Student não pareado ou Mann Whitney (amostras independentes), teste de Spearman (correlação) e teste do $\chi^{2}$ (proporção), com nível de significância $p<0,05$. Resultados: O GEP apresentou resultados inferiores significantes no desenvolvimento motor geral $(91,32 \pm 10,68$ versus $97,14 \pm 9,06$; $p=0,010)$, nas áreas de motricidade fina $-Q M 1(93,21 \pm 21,59$ versus $103,1 \pm 13,12 ; p=0,0138)$ e motricidade global - QM2 $(90,31 \pm 23,54$ versus $112,6 \pm 16,07 ; p<0,0001)$. Observou-se correlação positiva significativa entre o nível de atividade física e o desenvolvimento motor no GEP, nas áreas de equilíbrio - QM3 (0,35080; $p=0,0125)$ e organização espacial - QM5 (0,41820; $p=0,0025)$. Conclusão: O GEP, quando comparado com o GNP, demonstrou resultados inferiores no quociente motor geral (QMG), QM1 e QM2.

Palavras-chave: aptidão física; desenvolvimento infantil; habilidades motoras; obesidade infantil.

\begin{abstract}
Introduction: In school-age children, differences in weight and motor skills are still controversial. Objective: To analyze the relation between motor development, level of physical activity, and body mass index (BMI) in children with overweight. Methods: 85 students of both genders, aged 6 to 10 years, living in Presidente Prudente, São Paulo, Brazil, were evaluated. The instruments used were: the classification of BMI/age, the Motor Development Scale and the Modified and Adapted Questionnaire of Level of Physical Activity. The sample was divided into two groups: the overweight group (OWG) was composed of 50 students with nutritional diagnosis of overweight and obesity; the normal weight group (NWG) was composed of 35 eutrophic students. The following tests were used for statistical analysis: Shapiro Wilk (data normality), Student's t test or Mann-Whitney (independent samples), Spearman's test (correlation) and $\chi^{2}$ test (proportion), with significance level set at $p<0.05$. Results: The OWG had significantly lower results for general motor development (91.32 \pm 10.68 versus $97.14 \pm 9.06 ; p=0.010)$, fine motricity (93.21 \pm 21.59 versus $103.1 \pm 13.12 ; p=0.0138)$ and gross motricity $(90.31 \pm 23.54$ versus $112.6 \pm 16.07 ; p=0.0001)$. A significant positive correlation was found between level of physical activity and motor development in OWG subjects in the domains balance $(0.35080 ; p=0.0125)$ and spatial organization (0.41820; $p=0.0025)$. Conclusion: When OWG is compared to NWG, results are inferior for general motricity, fine motricity, and gross motricity.
\end{abstract}

Keywords: physical fitness; child development; motor skills; pediatric obesity. 


\section{INTRODUÇÃO}

A contínua mudança do comportamento motor que ocorre durante o ciclo da vida de um indivíduo é o resultado da interação entre fatores genéticos, biológicos e ambientais, sendo denominado desenvolvimento motor ${ }^{1}$. Esse processo dinâmico ocorre devido ao surgimento de novas formas de movimento ${ }^{2}$.

$\mathrm{O}$ impacto da atividade física ou da aquisição de habilidades motoras é especialmente pronunciado em crianças. Os comportamentos ativos constroem um repertório motor suficientemente adaptado para contextos específicos de movimento ${ }^{3}$. No entanto, há várias condições ambientais ou biológicas que colocam em risco o curso normal do desenvolvimento motor ${ }^{4}$. Dentre essas condições pode-se citar a obesidade ${ }^{5}$, que é uma doença originada a partir de uma combinação genética e fatores ambientais. Entre os fatores ambientais, encontram-se o sedentarismo e a alta oferta de alimentos ${ }^{6}$.

Globalmente, há crescente prevalência de sobrepeso e obesidade tanto em países desenvolvidos quanto em desenvolvimento. A taxa de obesidade triplicou nos países em desenvolvimento ao longo dos últimos 20 anos $^{7}$. No Brasil se observa um aumento progressivo na prevalência de excesso de peso $^{6}$, que pode ser resultado da redução da prática de atividade física entre crianças nas duas últimas décadas ${ }^{8,9}$.

Nesse contexto, Stodden et al. ${ }^{10}$ apresentaram um modelo conceitual que hipotetiza as relações entre atividade física, habilidade motora e obesidade. O qual demonstra importância mútua entre desenvolvimento motor e atividade física, em que ambos influenciam o estado nutricional, observando-se, dessa forma, que a junção do baixo desenvolvimento motor e nível de atividade física ocasionam a obesidade.

Ao investigar a relação entre o estado nutricional de crianças com diferentes perfis de competência motora e diferenças entre os níveis de atividade física, De Meester et al. ${ }^{5}$ concluíram que o sobrepeso e a obesidade estavam relacionados com a baixa competência motora real e percebida e com menor nível de atividade física. Marmeleira et al. ${ }^{11}$ encontraram que as crianças com peso normal apresentaram maior competência motora do que aquelas com sobrepeso ou obesidade.

Em contrapartida, Duncan et al. ${ }^{12}$ observaram que meninas com menor desenvolvimento motor têm maior índice de massa corporal (IMC) e porcentagem de gordura corporal, o que não foi evidenciado em meninos. Catenassi et al. ${ }^{13}$ avaliaram a relação entre a habilidade motora grossa com o IMC em crianças de quatro a seis anos de idade e não obtiveram resultados que comprovem relação entre ambos. Obtendo o mesmo resultado, Miranda et al. ${ }^{14}$ concluíram que a composição corporal não influencia a habilidade motora, e sim a estimulação ambiental. Pazin et al. ${ }^{15}$ apontam que crianças obesas não têm estimulação motora adequada ao seu desenvolvimento, fato que pode indicar menor incentivo às práticas de atividade física e/ou pela exclusão que grande parte delas sofre em âmbito escolar.

Observa-se que quanto maior o envolvimento das crianças em comportamentos ativos, mais elas construíram um repertório motor suficientemente adaptado para específicos contextos de movimento. Em contraste, a atividade física limitada pode contribuir para deficiência na coordenação motora. Intervenções na atividade física em crianças demonstraram ter impacto positivo na autoestima, especialmente pronunciado nas crianças obesas ${ }^{16}$.

Diferenças relacionadas ao peso e às habilidades motoras em crianças em idade escolar ainda apresentam controvérsias ${ }^{5,8,11-14}$. Sendo assim, o presente estudo diferencia-se dos citados e se mostra válido, pois teve como objetivo analisar a relação entre IMC, desenvolvimento motor e nível de atividade física em crianças com excesso de peso.

\section{MÉTODOS}

Realizamos um estudo transversal e observacional. Participaram deste estudo 85 crianças de ambos os sexos, com idades de seis a dez anos, regularmente matriculadas em cinco escolas municipais do ensino fundamental de Presidente Prudente, São Paulo. Essas cinco escolas foram selecionadas pela secretaria municipal de educação, sendo localizadas em diferentes zonas da cidade: zonas norte, sul, leste, oeste e centro, não privilegiando as possíveis diferenças socioeconômicas ou culturais.

A inclusão das crianças no estudo iniciou-se pela assinatura do Termo de Consentimento Livre e Esclarecido (TCLE) pelos responsáveis e do Termo de Assentimento (TA) por elas próprias. Foram excluídas as crianças que apresentaram alguma doença previamente diagnosticada por meio de laudos pertencentes às escolas selecionadas, tais como hipertensão arterial sistêmica, diabetes mellitus, afecções do sistema respiratório e nervoso.

Para realizar a mensuração do peso foi utilizada uma balança digital Plenna ${ }^{\circledR}$ com capacidade máxima de 160 kg e resolução de 100 gramas. O indivíduo com o uniforme escolar (shorts e camiseta) foi posicionado em pé no centro da plataforma da balança, com os pés descalços e com os braços posicionados ao longo do corpo.

$\mathrm{Na}$ avaliação da altura, que foi mensurada em metros (m), foi utilizada uma fita métrica com resolução de $0,1 \mathrm{~cm}$ que estava fixada em parede lisa. O indivíduo foi colocado em posição ortostática com os pés descalços e unidos, com as regiões occipital, cintura escapular, cintura pélvica e posterior do calcanhar em contato com a parede, com a cabeça permanecendo no plano horizontal de Frankfurt ${ }^{17}$.

Após determinar o IMC, utilizou-se a classificação de acordo com o Sistema de Vigilância Alimentar e Nutricional (SISVAN) ${ }^{18}$ Essa estratificação tem o objetivo de detectar situações de risco nutricional pelos seguintes critérios:

- Percentil <-0,1=magreza acentuada;

- Percentil $>0,1$ e $<3=$ magreza;

- Percentil $>3$ e $<85=$ eutrofia;

- Percentil $>85$ e $<97=$ sobrepeso;

- Percentil $>97$ e $<99,9=$ obesidade;

- Percentil $>99,9=$ obesidade grave 
O instrumento de avaliação do desenvolvimento motor utilizado foi a Escala de Desenvolvimento Motor (EDM) de Rosa Neto et al. ${ }^{19}$, a qual é indicada para avaliar crianças com idade entre 2 e 11 anos, sendo possível obter o perfil motor de uma forma mais abrangente, já que determina a idade motora (IM), o quociente motor geral (QMG), e também analisa e determina os quocientes das seguintes áreas do desenvolvimento: motricidade fina (QM1), motricidade global (QM2), equilíbrio (QM3), esquema corporal/ rapidez (QM4), organização espacial (QM5), linguagem/organização temporal (QM6), além de avaliar a lateralidade.

A complexidade da tarefa realizada aumentou de acordo com o aumento da idade. Os participantes foram avaliados a partir do teste correspondente à sua idade cronológica em cada elemento da área avaliada neste estudo e terminaram a avaliação quando não desempenharam corretamente a tarefa proposta. A idade correspondente à última tarefa desempenhada corretamente pela criança caracteriza-se por IM. Essa escala também permite a análise da relação entre a IM e a idade cronológica (IC), denominado quociente motor (QM). É por meio do QM que se classifica o desenvolvimento motor de cada sujeito em: muito superior (130 ou mais), superior (120 a 129), normal alto (110 a 119), normal médio (90 a 109), normal baixo (80 a 89), inferior (70 a 79) ou muito inferior (69 ou menos).

Para analisar o nível de atividade física desses escolares foi aplicado o Questionário de Avaliação da Atividade Física e do Sedentarismo em Crianças e Adolescentes, sugerido por Silva ${ }^{20}$. Após as crianças responderem a esse questionário, por meio de uma entrevista, elas foram classificadas de acordo com a frequência e a intensidade dos exercícios realizados: indivíduos inativos (que não realizam nenhuma atividade física); inadequadamente ativos (que realizam atividade física duas vezes ou menos semanalmente ou com duração inferior a uma hora); ativos (que realizam atividade física três vezes ou mais semanalmente e com duração superior a uma hora).

O estudo iniciou após ser aprovado pelo Comitê de Ética em Pesquisa (CEP) e pelo Comitê Assessor de Pesquisa Institucional (CAPI) da Universidade do Oeste Paulista (UNOESTE), sob o Protocolo de CAAE no 39744314.4.0000.5515.

Foram feitas avaliações de forma individualizada na própria escola que o participante frequentava. Inicialmente, foram coletadas informações relacionadas ao sexo, idade, peso, altura e circunferência abdominal. Após, foi determinado o IMC/idade, sendo utilizada a classificação de acordo com o SISVAN $^{18}$, e os escolares foram alocados por conveniência em dois grupos: grupo com excesso de peso (GEP) e grupo com normopeso (GNP). Foi aplicado também a EDM e o Questionário do Nível de Atividade Física. As avaliações foram realizadas na própria escola em que o participante estuda, no horário de aula, em um espaço disponibilizado pela direção da escola (sala ou quadra esportiva).

Após o término da coleta de dados foi feita uma análise estatística utilizando o software estatístico Graph Pad Prism. Para análise de normalidade dos dados foi utilizado o teste de Shapiro Wilk.
Para análise das amostras independentes foi utilizado o teste $t$ de Student não pareado ou Mann Whitney, de acordo com a normalidade dos dados. Para as análises de correlação foi utilizado o teste de Spearman. E as análises de proporção foram feitas por meio de tabela de contingência e analisadas pelo teste do $\chi^{2}$. O nível de significância utilizado foi de $5 \%$.

\section{RESULTADOS}

Foram avaliadas 85 crianças, sendo 50 do GEP e 35 do GNP, com média de idade cronológica de 8,26 $\pm 1,46$ anos. Como a EDM analisa a idade em meses, o GNP apresentou idade média de 96,80 meses, e idade motora média de 91,45 meses, o que representa uma idade motora negativa de 5,35 meses. O GEP apresentou idade média de 100,8 meses e idade motora média de 90,88 meses, representando assim uma idade motora negativa de 9,92 meses.

A Tabela 1 apresenta a classificação percentual do QMG. Nota-se que apenas o GEP apresenta classificações muito inferior e inferior.

Na Tabela 2 podemos observar as comparações entre o GNP e o GEP em relação à idade, ao IMC e à EDM. Na EDM obteve-se significância na comparação do QMG, QM1 e QM2, o que significa que, quando comparado com o GEP, o GNP apresenta melhor desenvolvimento motor nessas áreas.

$\mathrm{Na}$ Tabela 3 podem ser observadas as análises de correlação entre o desenvolvimento motor e o nível de atividade física dos grupos, sendo positiva no GEP entre nível de atividade física e as áreas de QM3 e QM5.

Tabela 1: Classificação do quociente motor geral para cada grupo

\begin{tabular}{|l|c|c|}
\hline Quociente motor geral & GNP (\%) & GEP (\%) \\
\hline Muito inferior & - & 2,0 \\
\hline Inferior & - & 12,0 \\
\hline Normal baixo & 14,3 & 28,0 \\
\hline Normal médio & 80,0 & 56,0 \\
\hline Normal alto & 5,7 & 2,0 \\
\hline Superior & - & - \\
\hline Muito superior & - & - \\
\hline
\end{tabular}

GNP: grupo com normopeso; GEP: grupo com excesso de peso.

Tabela 2: Comparação entre grupo com normopeso e grupo com excesso de peso

\begin{tabular}{|l|c|c|c|} 
& GEP & GNP & Valor $\mathbf{p}$ \\
\hline & $\mathbf{n = 5 0}(\mathbf{n} \pm \mathbf{D P})$ & $\mathbf{n}=\mathbf{3 5}$ & 0,173 \\
\hline Idade & $100,80 \pm 16,28$ & $96,80 \pm 19,51$ & $<0,0001^{*}$ \\
\hline IMC & $24,460 \pm 4,076$ & $15,65 \pm 1,94$ & $0,0102^{*}$ \\
\hline QMG & $91,32 \pm 10,68$ & $97,14 \pm 9,06$ & $0,0138^{*}$ \\
\hline QM1 & $93,21 \pm 21,59$ & $103,10 \pm 13,12$ & $<0,0001^{*}$ \\
\hline QM2 & $90,31 \pm 23,54$ & $112,60 \pm 16,07$ & 0,4969 \\
\hline QM3 & $101,50 \pm 17,95$ & $104,30 \pm 20,26$ & 0,3234 \\
\hline QM4 & $91,83 \pm 20,21$ & $96,08 \pm 13,35$ & 0,35 \\
\hline QM5 & $84,43 \pm 17,23$ & $89,02 \pm 20,74$ & 0,2691 \\
\hline QM6 & $75,71 \pm 17,61$ & $79,47 \pm 18,71$ & 0,3935 \\
\hline
\end{tabular}

GNP: grupo com normopeso; GEP: grupo com excesso de peso; *p<0,05; IMC: índice de massa corporal; QMG: quociente motor geral; QM1: motricidade fina; QM2: motricidade global; QM3: equilíbrio; QM4: esquema corporal/rapidez; QM5: organização espacial; QM6: organização temporal; n: número de crianças. 
Já para o GNP, observa-se comportamento negativo apenas para a área de organização espacial, o qual a correlação negativa expressa comportamento inverso entre o nível de atividade física e o escore (Tabela 3 ).

$\mathrm{Na}$ Tabela 4 verifica-se a associação entre os grupos quanto ao nível de atividade física. Observa-se que não houve diferenças estatísticas significativas entre eles e destaca-se uma porcentagem mais elevada de crianças consideradas ativas em relação às inadequadamente ativas e inativas. Quando observadas isoladamente as porcentagens de cada grupo e comparando-os, a maior discrepância pode ser notada na classificação inativa, na qual 30\% concentram-se no GEP, e na classificação ativa, na qual 54,3\% são do GNP.

\section{DISCUSSÃO}

Na classificação percentual do QMG, apenas o GEP apresentou classificações muito inferior e inferior. E na comparação do IMC e o desenvolvimento motor entre os grupos, observou-se que o GNP apresentou valores maiores do que o GEP, obtendo significância no desenvolvimento motor geral, QM1 e QM2, expressando que o GNP apresenta melhor desenvolvimento motor quando comparado com o GEP nessas áreas.

Poeta et al. ${ }^{21}$ também utilizaram a EDM, e assim como em nosso estudo, encontraram que crianças obesas apresentaram resultados inferiores significativos no desenvolvimento motor geral e QM2 em relação a crianças eutróficas. Além dessas áreas, observaram também resultados inferiores na QM3 e na QM4. Marramarco et al. ${ }^{22}$ justificam o desenvolvimento motor inferior de crianças obesas com os hábitos de vida da população.

Tabela 3: Análises de correlação entre os níveis de atividade física e Escala de Desenvolvimento Motor

\begin{tabular}{|l|c|c|c|c|}
\hline & \multicolumn{3}{|c}{ Nível de atividade física } \\
\hline & \multicolumn{2}{|c|}{ GEP } & \multicolumn{2}{c|}{ GNP } \\
\hline & $\mathbf{n}=\mathbf{3 5}$ \\
\hline QMG & Spearman $\mathbf{r}$ & Valor $\mathbf{p}$ & Spearman $\mathbf{r}$ & Valor $\mathbf{p}$ \\
\hline QM1 & 0,23390 & 0,1021 & $-0,09402$ & 0,5911 \\
\hline QM2 & 0,11900 & 0,4103 & $-0,02680$ & 0,8786 \\
\hline QM3 & 0,08089 & 0,5766 & 0,13510 & 0,4389 \\
\hline QM4 & 0,35080 & $0,0125^{*}$ & $-0,11810$ & 0,4992 \\
\hline QM5 & 0,05258 & 0,7169 & 0,08296 & 0,6357 \\
\hline QM6 & 0,41820 & $0,0025^{*}$ & $-0,43710$ & $0,0087^{*}$ \\
\hline & 0,11860 & 0,4122 & $-0,03988$ & 0,8201 \\
\hline
\end{tabular}

GNP: grupo com normopeso; GEP: grupo com excesso de peso; * $\mathrm{p}<0,05$; QMG: quociente motor geral; QM1: motricidade fina; QM2: motricidade global; QM3: equilíbrio; QM4: esquema corporal/rapidez; QM5: organização espacial; QM6: organização temporal; n: número de crianças.

Tabela 4: Comparação entre os níveis de atividade física entre os grupos

\begin{tabular}{|l|c|c|c|}
\hline Crianças & GEP (\%) & GNP (\%) & Valor $\mathbf{p}$ \\
\hline Inativas & 30,0 & 20,0 & 0,3002 \\
\hline Inadequadamente ativas & 24,0 & 25,7 & 0,8569 \\
\hline Ativas & 46,0 & 54,3 & 0,4521 \\
\hline
\end{tabular}

GNP: grupo com normopeso; GEP: grupo com excesso de peso.
De Meester et al. ${ }^{5}$ também investigaram a relação do IMC com o desenvolvimento motor de crianças, e assim como os estudos supracitados, concluíram que o sobrepeso e a obesidade estavam relacionados com a baixa competência motora real e percebida. Nunez-Gaunaurd et al. ${ }^{7}$ observaram que, além das crianças obesas demonstrarem maiores prejuízos na proficiência motora, também apresentam maior prejuízo em força e resistência comparadas às crianças com peso normal. Crianças obesas são propensas a terem menos passos por dia e a passarem mais minutos sedentárias. O estudo ainda elucida fatores modificáveis potenciais da obesidade infantil para ajudar a reduzir as disparidades de saúde em crianças provenientes de minorias.

Por sua vez, Chivers et al. ${ }^{23}$ examinaram se as pontuações de desempenho motor inferiores podem ser atribuídas à má coordenação, ou se as alterações de peso também podem afetar o desempenho do motor. Os sujeitos foram agrupados em peso normal, sobrepeso e obeso. Não houve diferenças significativas nos escores de desempenho motor global entre grupos. Os autores questionam a suposição de que o desempenho motor baixo está associado à obesidade e, segundo os mesmos, não se pode assumir que existe uma relação causal definitiva. Também levantam a questão de que alguns itens do teste podem não ser apropriados para uma população cada vez maior de indivíduos com sobrepeso.

O menor desempenho na QM2 apresentado pelo GEP pode ser justificado pelo fato das crianças com excesso de peso terem dificuldade em realizar os exercícios de correr e saltar, como demonstra o estudo de Berleze et al..$^{24}$. Poeta et al. ${ }^{21}$ relatam que o pior desempenho motor nos obesos pode relacionar-se a problemas ortopédicos, o que acarretaria alterações na mobilidade.

Castetbon et al. ${ }^{25}$ afirmam que as habilidades motoras das crianças são negativamente associadas à obesidade, não podendo haver essa correlação em crianças com quatro a seis anos de idade, sendo necessárias intervenções de atividade física destinadas a focar nas forças físicas das crianças obesas, e incentivar experiências de atividades bem-sucedidas. Reforçando esses resultados, Gentier et al. ${ }^{26}$ afirmam que a obesidade fornece um constrangimento para o desempenho motor grosso das crianças, sugerindo que crianças obesas têm dificuldades com a integração e o processamento da informação sensorial.

Outro estudo aponta que houve diferenças significativas na avaliação da QM1 das crianças obesas, que pode ser explicado pelo deslocamento anterior do centro de gravidade, o que gera alteração no equilíbrio, desordens posturais e dificuldades em adaptar-se a mudanças provenientes do meio externo ${ }^{27}$, sendo explicado também por Poeta et al. ${ }^{21}$, que, embora não tenham encontrado alterações nessa área, relatam que as crianças obesas tendem a apresentar tal alteração.

Em contrapartida, Catenassi et al. ${ }^{28}$ avaliaram a relação entre o desempenho em tarefas de habilidade motora grossa com o IMC em crianças de quatro a seis anos de idade e não obtiveram resultados que comprovem relação do IMC com o desenvolvimento motor. 
Contreira et al..$^{29}$ obtiveram o mesmo resultado e concluíram que a habilidade motora é influenciada pela estimulação ambiental e não pela composição corporal.

Cheng et al..$^{30}$ examinaram a influência do peso sobre habilidades motoras em crianças. Os resultados indicaram que um peso elevado contribui para um declínio nas habilidades motoras e não o inverso, sugerindo que os esforços de intervenção na obesidade infantil precoce podem ajudar a prevenir quedas na proficiência motora infantil, podendo produzir benefícios secundários de forma positiva, impactando nos níveis de atividade e de aptidão física das crianças.

Em relação à comparação do nível de atividade física com o desenvolvimento motor, observou-se uma correlação positiva no GEP entre o nível de atividade física e as áreas de QM3 e QM5, ou seja, quanto maior o nível de atividade física, melhor o escore nessas áreas. Corroborando este estudo, Morrison et al. ${ }^{31}$ encontraram que crianças com alta porcentagem de gordura e com alta performance motora eram fisicamente mais ativas. Esses resultados demonstram que uma criança com excesso de peso, quando estimulada de forma adequada, pode apresentar bom desenvolvimento motor.

Já para o GNP, observa-se comportamento inverso apenas na área de organização espacial, na qual a correlação negativa expressa que quanto maior o nível de atividade física, pior o escore nessa área. O que pode ser explicado pelo fato de que na realização desse teste as crianças necessitam de um bom entendimento sobre direita e esquerda, sendo válido ressaltar que a lateralidade está completa após os 9 ou 10 anos de idade ${ }^{32}$.

Bonvin et al. ${ }^{16}$ indicam que um programa de atividade física no cuidado da criança não leva à melhora nas habilidades motoras, e ressaltam a complexidade da implementação de uma intervenção de atividade física fora do ambiente de estudo.

O estudo de Bonvin et al. ${ }^{33}$ fornece informações sobre o status de peso e diferenças entre sexos em crianças de dois a quatro anos de idade que frequentavam creche. Nessa idade, as habilidades motoras globais e atividade física não diferiram de acordo com o status do peso. No entanto, os meninos apresentaram maior nível de atividade física do que as meninas.

A prática de atividade física independe somente do âmbito escolar. Embora esse seja parte importante no processo de desenvolvimento psicomotor, a criança recebe forte influência dos hábitos paternos e a prática de atividades deve ser incorporada por toda a família ${ }^{34}$.

Acredita-se que o número reduzido da amostra é uma limitação do estudo, visto que não foi uma amostra estatisticamente satisfatória. Vale ressaltar a importância de novos estudos comparando a relação entre IMC, desenvolvimento motor, nível de atividade física e demais variáveis (nível de atividade física dos pais, prematuridade, entre outros) para colaborar com o planejamento do tratamento da obesidade infantil e estimular a prática de exercícios físicos, prevenindo assim a própria obesidade infantil e seus agravantes no decorrer da vida.

Conclui-se que apesar da maior parte dos indivíduos do GEP apresentarem desenvolvimento motor normal, quando comparados com o GNP demonstraram resultados inferiores no QMG, QM1 e QM2. Porém, também foi observado no GEP que quanto maior o nível de atividade física, maiores foram os escores nas áreas de QM3 e QM5, demonstrando, assim, que aumentar a atividade física proporciona mais oportunidades para promover um melhor desenvolvimento neuromotor.

\section{REFERÊNCIAS}

1. Brito CML, Vieira GO, Costa MCO, Oliveira NF. Desenvolvimento neuropsicomotor: o teste de Denver na triagem dos atrasos cognitivos e neuromotores de pré-escolares. Cad Saúde Pública. 2011;27(7):1403-14.

http://dx.doi.org/10.1590/S0102-311X2011000700015

2. Kakebeeke TH, Caflisch J, Chaouch A, Rousson V, Largo $\mathrm{RH}$, Jenni OG. Neuromotor development in children. Part 3: motor performance in 3-to 5-year-olds. Dev Med Child Neurol. 2013;55(3):248-56

http://dx.doi.org/10.1111/dmcn.12034

3. Bonvin A, Barral J, Kakebeeke TH, Kriemler S, Longchamp A, Schindler C, et al. Effect of a governmentally-led physical activity program on motor skills in young children attending child care centers: a cluster randomized controlled trial. Int J Behav Nutr Phys Act. 2013;10:90

http://dx.doi.org/10.1186/1479-5868-10-90

4. Defilipo EC, Frônio JS, Teixeira MTB, Leite ICG, Bastos RR, Vieira MT, et al. Oportunidades do ambiente domiciliar para o desenvolvimento motor. Rev Saúde Pública. 2012;46(4):633-41. http://dx.doi.org/10.1590/S0034-89102012005000040

5. De Meester A, Stodden D, Brian A, True L, Cardon G, Tallir I, et al. Associations among Elementary School Children's Actual Motor
Competence, Perceived Motor Competence, Physical Activity and BMI: A Cross-Sectional Study. PLoS One. 2016;11(10):e0164600. http://dx.doi.org/10.1371/journal.pone.0164600

6. Nogueira-de-Almeida CA, Pires LAF, Miyasaka J, Bueno V, Khouri JMN, Ramos MLSR, et al. Comparison of feeding habits and physical activity between eutrophic and overweight/obese children and adolescents: a cross sectional study. Rev Assoc Med Bras. 2015;61(3):227-33.

http://dx.doi.org/10.1590/1806-9282.61.03.227

7. Nunez-Gaunaurd A, Moore JG, Roach KE, Miller TL, Kirk-Sanchez NJ. Motor proficiency, strength, endurance, and physical activity among middle school children who are healthy, overweight, and obese. Pediatr Phys Ther. 2013;25(2):130-8. http://dx.doi.org/10.1097/PEP.0b013e318287caa3

8. Raistenskis J, Sidlauskiene A, Cerkauskiene R, Burokiene S, Strukcinskiene B, Buckus R. Physical activity and sedentary screen time in obese and overweight children living in different environments. Cent Eur J Public Health. 2015;23(Suppl.):S37-43. http://dx.doi.org/10.21101/cejph.a4184

9. Pimenta TAM, Rocha R. A obesidade infantil no Brasil: um estudo comparativo entre a PNSN/1989 e a POF/2008-09 entre crianças de 5 a 9 anos de idade. FIEP Bul. 2012;82(Esp):1-5. 
10. Stodden DF, Goodway JD, Langendorfer SJ, Roberton MA, Rudisill ME, Garcia C, et al. A developmental perspective on the 20 role of motor skill competence in physical activity: an emergent relationship. Quest. 2008;60:290-306.

11. Marmeleira J, Veiga G, Cansado H, Raimundo A. Relationship Between Motor Proficiency and Body Composition in 6- To 10-YearOld Children. J Paediatr Child Health. 2017;53(4):348-53. http://dx.doi.org/10.1111/jpc.13446

12. Duncan MJ, Bryant E, Stodden D. Low fundamental movement skill proficiency is associated with high BMl and body fatness in girls but not boys aged 6-11 years old. J Sports Sci. 2017;35(21):2135-41.

http://dx.doi.org/10.1080/02640414.2016.1258483

13. Catenassi FZ, Marques I, Bastos CB, Basso L, Ronque ERV, Gerage AM. Relação entre índice de massa corporal e habilidade motora grossa em crianças de quatro a seis anos. Rev Bras Med Esporte. 2007;13(4):227-30. http://dx.doi.org/10.1590/S1517-86922007000400003

14. Miranda TB, Beltrame TS, Cardoso FL. Desempenho motor e estado nutricional de escolares com e sem transtorno do desenvolvimento da coordenação. Rev Bras Cineantropom Desempenho Hum. 2011;13(1):59-66. http://dx.doi.org/10.5007/1980-0037.2011v13n1p59

15. Pazin J, Frainer DES, Moreira D. Crianças obesas têm atraso no desenvolvimento motor. Rev Digital. 2006;11(101).

16. Bonvin A, Barral J, Kakebeeke TH, Kriemler S, Longchamp A, Schindler $\mathrm{C}$, et al. Effect of a governmentally-led physical activity program on motor skills in young children attending child care centers: a cluster randomized controlled trial. Int J Behav Nutr Phys Act. 2013;10:90. http://dx.doi.org/10.1186/1479-5868-10-90

17. Cabrera TFC, Correia IFL, Santos DO, Pacagnelli FL, Prado MTA, Silva TD, et al. Analisys of the prevalence of overweight and obesity and the level of physical activity in children and adolescents of a southwestern city of São Paulo. J Hum Growth Dev. 2014;24(1):67-72. http://dx.doi.org/10.7322/jhgd.73455

18. Brasil. Ministério da Saúde. Protocolos do Sistema de Vigilância Alimentar e Nutricional (SISVAN) na assistência à saúde. Brasília: Ministério da Saúde; 2008.

19. Rosa Neto F, Santos APM, Xavier RFC, Amaro KN. A importância da avaliação motora em escolares: análise da confiabilidade da escala de desenvolvimento motor. Rev Bras Cineantropom Desempenho Hum. 2010;12(6):422-7.

http://dx.doi.org/10.5007/1980-0037.2010v12n6p422

20. Silva OB. Questionários de avaliação da atividade física e do sedentarismo em crianças e adolescentes [Internet]. 2009 [cited on 2016 Nov 18]. Available from: http://departamentos.cardiol.br/ sbc-derc/revista/2009/45/pdf/Rev45-p14-p18.pdf

21. Poeta LS, Duarte MFS, Giuliano ICB, Silva JC, Santos APM, Rosa Neto F. Desenvolvimento motor de crianças obesas. Rev Bras Ciênc Mov. 2010;18(4):18-25.

22. Marramarco CA, Krebs RJ, Valentini NC, Ramalho MHS, Santos JOL, Nobre GC. Crianças desnutridas pregressas, com sobrepeso e obesas apresentam desempenho motor pobre. Rev Educ Fís UEM. 2012;23(2):175-82.

http://dx.doi.org/10.4025/reveducfis.v23i2.13002

23. Chivers $P$, Larkin D, Rose E, Beilin L, Hands B. Low motor performance scores among overweight children: Poor coordination or morphological constraints? Hum Mov Sci. 2013;32(5):1127-37. http://dx.doi.org/10.1016/j.humov.2013.08.006

24. Berleze A, Haeffner LSB, Valentini NC. Desempenho motor de crianças obesas: uma investigação do processo e produto de habilidades motoras fundamentais. Rev Bras Cineantropom Desempenho Hum. 2007;9(2):134-44.

25. Castetbon K, Andreyeva T. Obesity and motor skills among 4 to 6-year-old children in the United States: Nationally-representative surveys. BMC Pediatr. 2012;12:28. http://dx.doi.org/10.1186/1471-2431-12-28

26. Gentier I, D'Hondt E, Shultz S, Deforche B, Augustijn M, Hoorne S, et al. Fine and gross motor skills differ between healthy-weight and obese children. Res Dev Disabil. 2013;34(11):4043-51. http://dx.doi.org/10.1016/j.ridd.2013.08.040

27. Rafael CO, Mura MLBM, Fernani DCGL, Pires FHB. Análise do desenvolvimento motor em crianças com sobrepeso e obesidade através da escala de desenvolvimento motor de Rosa Neto. Temas Desenvolv. 2010;17(98):69-73.

28. Catenassi FZ, Marques I, Bastos CB, Basso L, Ronque ERV Gerage AM. Relação entre índice de massa corporal e habilidade motora grossa em crianças de quatro a seis anos. Rev Bras Med Esporte. 2007:13(4):227-30 http://dx.doi.org/10.1590/S1517-86922007000400003

29. Contreira AR, Capistrano R, Oliveira AVP, Beltrame TS. Indicadores de saúde em escolares: avaliação do estado nutricional e desempenho motor. Cinergis. 2013;14(1):13-17. http://dx.doi.org/10.17058/cinergis.v14i1.3533

30. Cheng J, East P, Blanco E, Kang Sim E, Castillo M, Lozoff B, et al Obesity leads to declines in motor skills across childhood. Child Care Health Dev. 2016;42(3):343-50 http://dx.doi.org/10.1111/cch.12336

31. Morrison KM, Bugge A, El-Naaman B, Eisenmann JC, Froberg K, Pfeiffer KA, et al. Inter-relationships among physical activity, body fat, and motor performance in 6-to 8-year-old Danish children. Pediatr Exerc Sci. 2012;24(2):199-209. http://dx.doi.org/10.1123/pes.24.2.199

32. Logan SW, Scrabis-Fletcher K, Modlesky C, Getchell N. The relationship between motor skill proficiency and body mass index in preschool children. Res Q Exerc Sport. 2011;82(3):442-8. http://dx.doi.org/10.1080/02701367.2011.10599776

33. Bonvin A, Barral J, Kakebeeke TH, Kriemler S, Longchamp A, Marques-Vidal $\mathrm{P}$, et al. Weight status and gender-related differences in motor skills and in child care-based physical activity in young children. BMC Pediatrics. 2012;12:23 http://dx.doi.org/10.1186/1471-2431-12-23

34. Vanelli CP, Novaes CA, Castro MR, Pereira HAC, Carvalho RS, Ferreira VN. Verificação e descrição do índice de sobrepeso de escolares da rede pública da cidade de Matias Barbosa. HU Rev. 2011;37(1):7-13. 